\title{
ENTWICKLUNG VON INTERAKTIVEN LERNMEDIEN MIT DER MACROMEDIA FLASH PROFESSIONAL 8 ZUM THEMA,DEUTSCHE GESCHICHTE"
}

\author{
Citra Doris Simorangkir \\ Laurensius Tampubolon \\ Hafniati
}

\begin{abstract}
AUSZUG
Das Ziel dieser Untersuchung ist es, um eine interaktiven Lernmedien mit der Macromedia Flash Professional 8 zum Thema „Deutsche Geschichte” zu erstellen.DieErstellungsuntersuchung werden in dieser Untersuchung angewendet. Der Prozess der Erstellung dem interaktiven Lernmedien mit derMacromediaFlashProfessional8zum Thema „Deutsche Geschichte” verwendetADDIE-Modell.Die Erstellungsphase besteht aus fünf Phasen. Die Phasen sind(1) Analyse, (2)Design,(3) Entwicklung, (4) Durchführung,(5) Bewertung. Die Daten in dieser Untersuchung sind informationen über 16 Bundesländer, Weimarer Republik, die Teilung Deutschlands, die Wiedervereinigung Deutschlands bei der Deutschen Geschichte.Die Datenquelle in dieser Untersuchung kommt aus das Buch Landeskunde Deutschland, Deutsche Geschichte erzählt von Manfred Mai, Artikel aus dem Internet und Youtube.Diese Untersuchung wird in der Bibliothek an der Fakultät für Sprachen und Kunst an der Staatlichen Universität von Medan durchgeführt. Die Ergebniss der Erstellung eine interaktiven Lernmedien mit der Macromedia Flash Professional 8 zum Thema „Deutsche Geschichte”, besteht aus 70 Seite und die Datei umfasst 686 MB (Megabyte), dann gibt es das Video, die Bilder und die Übungen. Nach der Erstellung wurde die interaktiven Lernmedien bei der Experten validiert oder überpruft, Basierend auf der Validierung ist die interaktiven Lernmedien sehr gut. Durch die Ergebnisse der Untersuchung wird erwartet, um unser Wissen zur Wisschenshaft über die interaktiven Lernmedien, vor allem Deutschlernenden anzureichen.

Schlüsselwörter:die interaktiven Lernmedien, Macromedia Flash Professional 8 , Deutsche Geschichte.
\end{abstract}

\section{EINLEITUNG}

Medien werden in zwei Arten unterteilt, nämlich Etymologie und Terminologie. Etymologisch ist „medien” ist der plural des Wortes “medium” von lateinischen "medius", was die mittlere bedeutet. Terminologisch erklärt Schraam (in Azhar, 2011:7), dass Medien ein Kommunikationsmittel sind, die für Lernzwecke genutzt werden können. Medien spielen eine wichtige Rolle im Lernprozess und sind einer der entscheidenden wichtige Faktoren beim Lernen, 
weil die Medien ein mittel sein können, Lernmaterialen zu liefern, Nachrichten zu kommunizieren und die Lernmotivation zu verbessern.

Rusman et al (2012:63) erklärt, dass die Art des Mediums, das als Lernmedium verwendet wird, visuelle Medien, audio Medien., audiovisuelle Medien, Mediengruppe, Medienobjekt und Computer-Medien sein können. Computer-Medien sind interaktive Lernmedien die als interessante und lustige lernmedien benutz werden können. Die interaktive Medium ist eine MedienNachrichten zwischen der Lehrern und der der Schülern, die die Kommunikation zwischen Mensch und Technick durch Systeme und Software ist (Tania in Website 2015). Das Erstellen von interaktiven Medien zum Lernen erfordert die Verfügbarkeit von Computergeräten. Diese Computergeräte sind Hardware und Software. Hardware, unter anderem ist die CPU, Tastatur, Monitor, Festplatte, CD-ROM und Drucker, während die Software eine ComputerProgrammiersprache ist. Das Software-Programm, das in dieser Forschung entwickelt wird, ist Macromedia Flash Professional 8 mit Anwendungen wie Video, Prozentsatz, Interaktive Animations, Bild und Ton als Lernmedium zu deutschen Geschichten.

Deutsche Geschichte ist eines der Fächer, die Deutschlernende um fünften semester erlernen. Das Lernen der deutschen Geschichte auf der Grundlage des Curriculums der Deustchprogramm Fakultät für Sprache und Kunst der Staatlichen Universität von Medan, das bei 16 Sitzungen in einem Semester stattfindet, besteht aus diesem Lernmaterialien: die Geschichte von 16 Bundesländer in Deutschland, dem Ersten Weltkrieg, der Weimarer Republik, dem Zweiten Weltkrieg, die Teilung Deutschland, dem Kalten Krieg, deutsche Wiedervereinigung und aktuelle Deutschland-Bedingungen (Lernplan Deutsche Geschichte). Dieses Thema hat eine Menge von Punkten, die gelernt werden. Daher brauchen neue Medien beim lernen der Deutsche Geschichte.

Im Deutschabteilung können Macromedia Flash Professional 8 als nues intraktive Lernmedium verwendet werden, wenn man die Deutsche Geschichte lernt.Am 26 Juli 2017 wurde eine Umfrage mit die Studenten in der Deutschabteilung gemacht. Das Ergebnis der Umfrage war, viele Deutschlernende 
haben noch Schwierigkeiten, die Deutsche Geschichte zu verstehen. Mithilfe von Macromedia Flash Professional 8 werden die Deutschlernende eher zum Lernen der Deutsche Geschichte kann leichter verstanden werden. Die Kriterien, bei der Nutzung des interaktiven Lernmediums zur deutschen Geschichte besitzen müssen, sind die Fähigkeit des Computerverständisses und die Auswahl der Lernressourcen, die Fähigkeit, die Lernstrategie und die Lernmethode der deutschen Geschichte zu entwerfen, und die Lernaktivitäten vorbereiten.

Basierend auf den oben genannten Problemen im Deutschabteilung wird „Entwicklung Von Interaktiven Lernmedien Mit Der Macromedia Flash Professional 9 Zum Thema Deutsche Geschichte”.

\section{THEORETISCHE UND KONZEPTUELLE GRUNDLAGEN}

\section{Das Entwicklungsmodell}

Sugyono (2009:3) sagt, dass sich die Entwicklung vertieft und das vorhandene Wissen erweitert. Es gibt viele Theorie zur Entwicklung von dem Erstellungmodell. Einige davon sind beispieleweise von Dick und Carey, Borg \& Gall, Kem, Morrison \& Ross oder von Plomp und ADDIE benutzt. Das ADDIE Modell ist ein Modell der Lehrentwürfe das von Reiser und Mollenda in den 1990er Jahren entwickelt wurde. Asrar (2013:118) beschreiben, dass das ADDIE Model als Anleitung beim Aufbau der Vorrichtung erfult und Trainingsprogramme effektive, dynamisch und unterstütz die Ausbildung. ADDIE ist ein Akronym für Analyse (Anaysis), Design (Design), Entwicklung (Development), Durchführung (Implementation), Bewertung (Evaluation).

\section{Der Begriff des Lernmedium}

Arsyad (2011:3) erklärt, dass das Wort Medien vom Lateinischen „Medius” stamm, was „Mittel”, „Vermittel”, oder „Begleiter” bedeutet. Heinnich (in Susilana, 2008:6) sagt, dass das Medium ein Kommunikationsmittel ist. Heinnich sagt auch, dass Fernseher, Computer, Diagramme, Bilder, Bücher und Magazine Beispiele für Medien sind.

Gagne ( in Arsyad, 2002:4) stellt fest, dass Lernmedien Werkzeuge beinhalten, die physisch verwendet werden, um den Inhalt von Lehrmaterialien zu vermitteln, die aus anderen Büchern, Tonbandgeräten, Bändern, Videokameras, 
Videorecordern, Filmen, Fotografien, Dias, Bildern, Grafiken, Fernsehen bestehen. Zainal Aqib (2014:60) erklärt, dass die Lernmedien etwas sind, das genutzt werden kann, um die Botschaft $\mathrm{zu}$ kanalisieren und den Lernprozess $\mathrm{zu}$ stimulieren. Der Einsatz von Lernmedien im Unterricht und Lernprozess kann neue Motivation und Interesse generieren, und auch psychologische Einflüsse auf Studenten haben (Arsyad, 2013:19).

Nach den obigen Meinungen kann zusammengefasst werden, dass Medien Mittel sind, die das Lernen erleichtern können. Mit Hilfe von Medien kann der Lernprozesss den Schülern Spaß machen. Dann sind die Schüler motiviert, fleißig zu lernen.

\section{Die Funktion der Lernmedium}

Nach Daryanto (2010:8) haben die Medien im Lernprozesse eine Funktion als Träger der Informationen von der Quelle (dem Lehrer) an den Empfänger (die Schüler) (vgl. Barus und Suratno 2015:17). Die Funktion des Lernmediums sind: a) Medien werden als Hilsmittel im Lernprozess eingesetzt, um eine effektive Lernsituation zu schaffen, b) Die Nutzung des Mediums hat das Ziel, Inhalte des Unterrichts $\mathrm{zu}$ integrieren, c) Die Lernmedien im Unterricht sind keine Vergnüngsmittel. Das bedeutet, dass sie den Lernprozess ergänzen, um das Interesse der Studenten zu wecken ( Sudjana, vgl. In Djamarah 2006:134)

Nach Sudjana und Rivai (in Djamarah 2006:134) sind die Vorteile des Lernens von Medien sowohl im Allgemeinen als auch im Besonderen als Lerninstrument für Lehrer und Lernende. Die Vorzüge der Lehrmedien sind also:

a. Die Lehre zieht mehr Schüler an, damit die Lernmotivation gesteigert wird.

b. Lernmaterialien sind klarer, was von den Schülern besser verstanden werden kann und ihnen ermöglicht, Lernziele zu erreichen

c. Lehrmethoden werden vielfältiger, nicht nur mündliche Kommunikation durch die Worte des Lehrers, so dass die Schüler nicht gelangweilt sind und der Lehrer nicht zu seht belastet wird. 
d. Die Schüler können mehr lernen, weil sie nicht nur die Beschreibung des Lehrers hören, sondern auch andere Aktivitäten nachgehen wie Beobachten, Durchführen, Demonstrieren, Spielen und so weiter.

Es kann also zusammengefasst werden, dass die Lernmedien zum einen die Lehrer dabei unterstützen, den Lernstoff einfacher zu erklären. Zum anderen können die Schüler den Lernstoff so leichter verstehen und werden dadurch für das Deutsche Geschichte begeistert und motiviert.

\section{Interaktive Lernmedien}

Jedes Lenmedium hat unterschiedliche Eigenschaften und Fähigkeiten, es ist erwähnenswert, um die Medien entsprechend den Bedingungen und Bedürfnissen zu wählen. Seels und Glasgow in Azhar (2002: 33-34), gruppieren die Medien in zwei breite Kategorien, nämlich traditionelle Medien und fortgeschrittene Technologie-Medien. Interaktive Medien beinhalten modernste technologische Medien. Eingeschlossen in der Medienkantentechnik, sind: (1) eine medienbasierten Telekommunikations, wie Telekonferenz, Fernunterricht, und (2) ein medialer Mikroprozessor, wie Beispielsweise computer assited intructions (CAI), Computerspiele, System Tutor Intelligenz, interaktive, hypermedia und Compact (Video) Disc.

Interaktive Lernmedien sind ein Lehr-Delivery-System, das VideoAufnahmematerial mit Computer-Steuerung präsentiert, für Lernenden nicht nur hören und sehen (Seels \& Glasgow in Azhar, 2002: 36). Ouda in Sukoco (2014: 221), erklärt, im Allgemeinen gibt es mehrere Kriterien, die verwendet werden können, um die Qualität eines interaktiven Mediums zu bewerten, unter anderem:

1. Einfache Navigation, was bedeutet, dass die Medien im Lernprozess einfach zu bedienen sind.

2. Inhalt der Kognition und inhalt um die Graduiertenkompetenzstandards zu erreichen.

3. Wissen und Präsentation von Informationen, Fokus des Wissens und Klarheit der Informationen.

4. Medienintegration, Kompatibilität zwischen Informationen und Illustrationen. 
5. Funktion als Ganzes, die Kompatibilität zwischen Medienleistung mit Lernzielen.

\section{Macromedia FlashProfessional 8}

Macromedia Flash ist beliebt, um Anwendungsmedien zu erstellen, weil wir Flash-Animationen erstellen können, Spiele, Ton, und so weiter. Macromedia Flash auf der neuesten Ausgabe ist am 13. September 2005 in zwei Arten von verteilt. Die erste ist Macromedia flash Basic 8 und der zweite ist Macromedia Flash Professional 8 (Kusrianto, 2006:1).

Anggara (2008:2) erklärt, Macromedia Flash Professional 8 ist eine Software-Version der Macromedia.inc in Form von Grafik und Animationsprogramm. Nach Wahyono (2006:1) ist Macromedia Flash 8 eine Anwendung, die für die Gestaltung und Präsentationsvorrichtung, Veröffentlichungen oder andere Anwendungen machen, die die Verfügbarkeit von Mitteln zur Interaktion mit den Nutzern erfordern. Die Projekte mit Flash erstellten sind Text, Bilder, einfache Animationen, Videos oder andere Spezialeffekte.

\section{Thema „Deutsche Geschichte"}

Das Lernen der deutschen Geschichte auf der Grundlage des Curriculums der Deustchprogramm Fakultät für Sprache und Kunst der Staatlichen Universität von Medan, das bei 16 Sitzungen in einem Semester stattfindet, besteht aus diesem Lernmaterialien: die Geschichte von 16 Bundesländer in Deutschland, dem Ersten Weltkrieg, der Weimarer Republik, dem Zweiten Weltkrieg, die Teilung Deutschland, dem Kalten Krieg, deutsche Wiedervereinigung und aktuelle Deutschland-Bedingungen (Lernplan Deutsche Geschichte).

Die Auswahl der Unterrichtsmaterialien zu deutschen Geschichte in dieser Studie wurden in randomisierten, die 4 sitzungen nämlich: 16 Bundesländer, Weimarer Republik, die Teilung Deutschland und die Vereinigung Deutschland.

\section{Die konzeptuelle Grundlage}

Deutsche geschichte ist eines der fächer, die Deutschlernende um fünften semester erlernen. Dieses Thema hat eine Menge von Punkten, die gelernt werden. Daher brauchen neue Medien beim lernen der Deutsche Geschichte. Im diesem 
Fall können Macromedia Flash Professional 8 als neues intraktive Lernmedium verwendet werden, wenn man die Deutsche Geschichte lernt. Viele Deutschlernende haben noch Schwierigkeiten, die Deutsche Geschichte zu verstehen. Mithilfe von Macromedia Flash Professional 8 werden die Deutschlernende eher zum Lernen der Deutsche Geschichte kann leichter verstanden werden. Da in der Macromedia Flash Professional 8 gibt es Video, Prozentsatz, Interaktive Animations, Bild und Ton als Lernmedium.

Durch die Verwendung von Macromedia Flash Professional 8 als interaktiven Lernmedien wird in die Deutschlernende interessiert und motiviert, um Deutsche Geschichte zu lernen. Das Material, das in dieser Forschung diskutiert werden soll ist: 16 Bundesländer, Weimarer Republik, die Teilung Deutschlands, die Vereinigung Deutschlands in der Deutschen Geschichte sind.. Das Entwicklungsmodell, das in dieser Forschung verwendet wird, ist das ADDIE-Entwicklungsmodell.

\section{UNTERSUCHUNGSMETHODOLOGIE}

Dieser Untersuchung ist eine Erstellungsuntersuchung. Es wird die Software Macromedia Flash Professional 8 als interaktives Lernmedium erstellt und dabei die Modell von ADDIE benutzt.

Die Daten dieser Untersuchung sind informationen über 16 Bundesländer, Weimarer Republik, die Teilung Deutschlands, die Vereinigung Deutschlands bei der Deutschen Geschichte Die Datenquelle sind aus das Buch Landeskunde Deutschland, Deutsche Geschichte erzählt von Manfred Mai, Artikel aus dem Internet und Youtube.

Diese Untersuchung wird in der Bibliothek der Fakultät Sprache und Kunst durchgeführt.

Die ADDIE Modell (Analyse-Design-Entwicklung-Durchführung-Bewertung).

Das ADDIE Modell, Das Folgende sind die Schritte des ADDIE-Modells:

1. Analyse (Anaysis)

a) Die Informationen über das Lernen des Studenten Bedürfnisse

b) Die Lernmedien im deutsche Geschichte unterricht gesammelt

c) Das Lernen der Schüler Anlage 
d) Vollständigkeit Bedingungen

e) das Lernen der Schüler zu unterstützen

2. Design (Design)

a) Die Autor verwendet die Anwendung Macromedia Flash Professional 8 in der Gestaltung.

b) Es wird das Konzept gemacht, zum Beispiel das Konzept des Design der interaktiven Lernmediums, Thema, Hintergrund, Bilder, Stimme und Musik.

3. Entwicklung (Development)

a) Prozess der Realisierung der Design in der Realität.

b) die Vorbereitung aller Mittel und Materialien, die für dei Erstellung der interaktive lernmedium verwendet werden sollen

c) die Computer mit Macromedia Flash Professional 8 anwendung

d) Danach wird das Lernmedium zum Thema „Deutsche Geschichte” erstellt.

4. Durchführung (Implementation)

a) ein konkreter Schritt ein Lernsystem zu implementieren, das erstellt wird.

b) alles das entwickelt wurde, installiert ist oder in einer solchen Art und Weise angebracht, die Rollen oder Funktionen eingestellt umgesetzt werden.

5. Bewertung (Evaluation)

a) eine validierung wird durchgeführt, um herauszufinden, ob das Lernmedium gut und interessant ist oder noch verbessern werden sollte.

b) Die Validierung wird von einem Experte durchgeführt.

\section{ERGEBNIS DER UNTERSUCHUNG}

Dieses Kapital handelt von den Untersuchungsergebnissen. In diesem Kapitel werden die Ergebnisse der Entwicklung eines interaktiven Lernmediums mit der Macromedia Flash Professional 8 zum Thema „Deutsche Geschichte” erklärt. Jeder Schritt hat ein eigenes Untersuchungsergebnis. Die Schritte der Untersuchung und die Ergebnissen werden in diesem Kapitel erklärt. 


\section{Der Prozess der Entwicklung}

In dieser Untersuchung wird das Untersuchungmodell von ADDIE benutzt. Dieses Modell besteht aus fünf Phasen, nämlich (1) Analyse (Anaysis), (2) Design (Design), (3) Entwicklung (Development), (4) Durchführung (Implementation), (5) Bewertung (Evaluation). In diesem Kapitel wird jede Stufe mit ihrem Ergebnis erklärt.

1. Analyse (analisys)

Diese ist die erste Phase, um ein interaktives Lernmedium mit der Macromedia Flash Professional 8 zum Thema „Deutsche Geschichte” zu erstellen. In dieser Phase der Beobachtung werden Informationen über das Lernen der Deutschlernenden, ihrer Bedürfnisse und die Lernmedien im für das Fach Deutsche Geschichte gesammelt. Zunächst wird eine Umfrage gemacht. Das Ergebnis der Umfrage war, dass die Bedürfnisse den Deutschlernenden ist, was die Medien nicht finden, dass die Deutschlernenden ein interaktiven Lernmedium zum Thema „Deutsche Geschichte” brauchen. Deshalb ist es sehr wichtig, ein interaktiven Lernmedien zu erstellen.

Die Auswahl der Unterrichtsmaterialien zu deutschen Geschichte in dieser Studie wurden in randomisierten auf Lernplan Deutsche Geschichte (RPP) . Es gibt fünft Daten. Das sind : (1) 16 Bundesländer, (2) Weimarer Republik, (3) die Teilung Deutschland, (4) die Vereinigung Deutschland, (5) Übungen.Das Thema „Deutsche Geschichte” wird von Studenten im fünften Semester gelernt.Die Datenquelle dieses Thema wird vondas Buch Landeskunde Deutschland, Deutsche Geschichte erzählt von Manfred Mai,Internet und Youtube genommen.

\section{Design (Design)}

In dieser zweiten Phase wird die Designphase durchgefuhrt, die auf von der ersten Phase basiert. Die Präsentation bei der Macromedia Flash Professional 8 wird mit den umfassenden Erklärungen und mit den Wörtern, Bildern und Videos von dem Thema „Deutsche Geschichte” dargestellt.In dieser Phase wird das Konzept des interaktiven Lernmedien mit der Macromedia Flash Professional 8 entworfen, um ein interessantes Lernmediem zu erstellen. 
Das Konzept des wünschwerten Design für diese interaktiven Lernmedien sind:

1. Das Hauptmenü

Das Haptmenü ist der wichtige teil für die interaktiven Lernmedien. In dieser interaktivne Lernmedien gibt es einige Menü und in einem menü kann das Submenü vorhanden sein. Das Submenü ist der Teil des Hauptmenüs, die aus jeder Kategorie erläutet wird. Das Menü besteht aus einige Teile, das sindProfil, Vorwort, das Hinweis, das Thema, Übungen und Literaturverzeichnis,. Unten werden die Menü mit eigenen Submenü erklärt.

a) Das Hinweismenü

Die Submenü des Hinweis Menüs sind das Verwendungsziel des Interaktiven Lernmediums und der Verwendungszweck des interaktiven Lernmediums.

b) Das Thema

Die Submenü des Thema sind 16 Bundesländer, Weimarer Republik, Die Teilung Deutschland und Wiedervereinigung Deutschland.

3. Entwicklung (Development)

Der dritte Schritt in dieser Untersuchung ist die Entwicklung des Produkts. In dieser Phase wird der Gebrauch der Macromedia Flash Professional 8 beschreiben. Bevor das lernmedium mit der Macromedia Flash 8 entworfen wird, werden die Schritte vorgestellt, die bei der Gestaltung des Design des Lernmediums berücksichtig werden müssen, nämlich:

a) Die Startseite der Macromedia Flash Professional 8

b) Der Hintergrund

c) Die Taste

d) Das Bild

e) Der Text

f) Das Video

g) Die Übungen

h) Die Datei speicheren

4. Durchführung (Implementation) 
Das Konzept, das in der dritte Phase geplant wird, wird in dieser Phase verwirklich. Die Bilder, Texte und Videos werden in dem Material zum Thema „Deutsche Geschichte” implementiert.

5. Auswertung (Revision)

Das ist der letzte Schritt, um ein interessante Lernmedium zu erstellen. In dieser Phase wurde schon das interaktiven Lernmedium mit der Macromedia Flash Professional 8erstellt. Es wird eine Validierung durchgeführt. So kann herausgefunden werden, ob das interaktiven Lernmedium mit der Macromedia Flash Professional 8 schon gut genug ist oder noch verbessert werden sollte. Nach der Validierung wird das Lernmedium mit der Macromedia Flash 8 verbessert. Dieses Lernmedium wird von der Experte geprüft. Der Experte ist ein Dozent, die viel über Medium weisst.

Der Experte hat das interaktiven Lernmedien mit der Macromedia Flash Professional 8 schon korrigiert. Danach hat der experte das interaktiven Lernmedien benotet: Von 10 Kategorien der Aspekt der Evaluation gibt der Experte die Note 4 für die acht Aspekt, nämlich wie die interaktiven Lernmedien, das Thema, der Gestaltung, inhalt dem erstellen interaktiven Lernmedien, die Wörter im der Macromedia Flash Professional 8, die Bilder, das video, die Leichtigkeit des Verständnisses. Die Note 4 bedeutet sehr gut. Der Experte gibt die Note 3 für die verwendung die Taste und Auswahl von Hintergrundfarbe. Die Note 3 bedeutet gut,sodass die Note des interaktiven Lernmedien mit der Macromedia Flash Professional 8 von dem Experte ist 90,50. Basierend auf der obigen meinung kann festgehalten werden, dass das interaktiven Lernmedien mit der Macromedia Flash Professional 8 interessant gestaltet ist und besonderes gut für das Thema „Deutsche Geschichte” ist.

\section{Der Ergebniss des Produkts}

Das Ergebnis der Untersuchung ist eine interaktiven Lernmediums mit der Anwendung Macromedia Flash 8. Diese interaktiven Lernmedien besteht aus 69 Seite und die Datei umfasst 782 MB (Megabyte). Die interaktiven Lernmediums kann mithilfe des GOM Players und SWF geöffnet warden. Unten werden die Ergebisse des interaktiven Lernmediums erklärt. 

a) Die Anzeige im Hauptmenü
b) Die Anzeige der Profilmenü
c) Die Anzeige Vorwortmenü
d) Die Anzeige das Hinweismenü
e) Die Anzeige das Verwendungsziel interaktiven Lernmedien
f) Die Anzeige das Verwendungszweck das interaktiven Lernmedien
g) Die Anzeige das Themamenü
h) Die Anzeige das Themainhalts
i) Die Anzeige der Übungen
j) Anzeige der Literaturverzeichnis

\section{Die Diskussion}

Basierend auf den Ergebnissen des interaktiven Lernmedien mit der Macromedia Flash Professional8 wurde zusammengefass, dass die Daten in dieser Untersuchung Bilder und Wörter aus dem Artikel aus Internet und Youtube genommen werden. In dieser Unteruchung wird die ADDIE-Modell benutz. Diese Modell besteht aus fünft Phasen. Die Phasen sind : (1) Analyse (Analysis), (2) Design (Design), (3) Entwicklung (Development), (4) Durchführung (Implementation), (5) Bewertung (Evaluation). In diesem Kapitel wird jede Stufe mit ihrem Ergebniss erklärt. In der Analysephase wurde eine Umfrage durchgeführtInformationen über das Lernen der Deutschlernenden, ihrer Bedürfnisse und die Lernmedien im Deutschgeschichte unterricht gesammelt.Das Ergebnis der Umfrage war, die Bedürfnisse den Deutschlernenden ist, was die Medien nicht finden, dass die Deutschlernenden ein interaktiven Lernmedium zum Thema „Deutsche Geschichte” brauchen. Deshalb ist es sehr wichtig, ein interaktiven Lernmedien zu erstellen.Die Auswahl der Unterrichtsmaterialien zu deutschen Geschichte in dieser Studie wurden in randomisierten auf Lernplan Deutsche Geschichte (RPP) . Es gibt fünft Daten. Die Datenquelle dieses Thema wird von das Buch Landeskunde Deutschland, Deutsche Geschichte erzählt von Manfred Mai,Internet und Youtube genommen.

In dieser zweiten Phasen wird die Designphase durchgeführt, die auf dem Ergebnis von der ersten Phase basiert. Die interaktiven Lernmedien bei der 
Macromedia Flash Professional 8 wird mit den umfassenden Erklärungen und mit den Würten, Bildern und Videos von dem Thema „Deutshe geschichte” aus dem Buch, Artikel aus Internet und Youtube dagestellt. In dieser Phase wird das Konzept des interaktiven Lermedien mit der Macromedia Flash Professional 8 entworfen, um ein interessantes interaktiven Lernmedien zu erstellen. Das Design wird an das Thema „Deutshe geschichte” angepasst, die in der Macromedie Professional 8 integriert werden. Das interaktiven Lernmedien wird interessantes, wenn Bilder, Wörter und Videos integriert werden. In dieser interaktiven Lernmedien hat fünft Hauptmenü, sie sind (1) Profil , (2) Vorwort , (3) das Hinweis (4) das Thema und (5) Übungen und (6) Literaturverzeichnis.

Die dritte Phase umfasst die Entwicklung des produkt. In der Entwicklungphase wird die Bilder, Texten und Videos werden in dem Material zum Thema „Deutshe Geschichte” realisiert. Während der Entwicklung interaktiven Lernmedien mit der Macromedia Flash Professional 8 fanden die Forscher eine Dinge, die berücksichtigt werden sollten, wie schreiben einen Actions Script, kann dies zu Fehlern führen, Daher muss das schreiben Actions Script gründlich und vollstandig sein, das Video muss eine bestimmte Größe haben, es muss das Video zuerst konvertieren und Dateien sollten in einem einziegen Ordner gespeichert werden. Das Thema „Deutshe geschichte” hat schon in der Macromedia Flash Professional 8 realisiert.In der Durchführung (Implementation)wird die Bilder, Texten und Videos werden in dem Material zum Thema „Deutsche Geschichte” implementiert (realisiert). Das Thema „Deutsche Geschichte" hat schon in der Macromedia Flash Professional 8 implementiert,Danach wird in der Revisionsphase schon das interaktiven Lernmedien mit der Macromedia Flash Professional 8 erstellt. Es wird einen Validierung durchgeführt. Der Experte hat interaktiven Lernmedium mit der Macromedia Flash Professional 8 schon korrigiert. Dann hat der Experte das interaktiven Lernmedien benotet : von 10 Kategorien der Aspekt der Evaluation gibt der Experte die Note 4 für die acht Aspekt, Der Experte gibt die Note 3 für die zwei Aspekt, sodass die Note des interaktiven Lernmedien mit der Macromedia Flash Professional 8 von dem Experte ist 90,50 (sehr gut). 
Basierend auf die obigen Basierend auf die obigen Erklärung kann zusammengefasst werden, dass die Erstellung des interaktiven Lernmediums mit der Macromedia Flah Professional 8 zum Thema „,Deutsche Geschichte” mit der ADDIE Theorie sehr gut ist. Sodass die Dozenten oder die Studenten das interaktiven Lernmedium benutzen kann.

\section{SCHLUSSFOLGERUNG}

Nachden Untersuchungsergebnissen fallen die Schlussfolgerungen folgendermaßen aus:

1. Der Prozess der Erstellung eine interaktiven Lernmedien mit der Macromedia Flash Professional 8 zum Thema „Deutsche Geschichte” über 16 Bundesländer, Weimarer Republik, die Teilung Deutschland und Wiedervereinigung Deutschland besteht aus der Erklärung der Phasen von ADDIE-Modell.Darunter sind: (1) Analyse (Anaysis), (2) Design (Design),

(3)Entwicklung (Development), (4) Durchführung (Implementation), (5) Bewertung (Evaluation).

2. Das Ergebnis der Erstellung eine interaktiven Lernmedien mit der Macromedie Flash Professional 8 zum Thema „Deutsche Geschichte” sind:

a. Dieser Untersuchung hat eine interaktiven Lernmedien mit der Macromedia Flash Professional 8 zum Thema „Deutsche Geschichte" erstellt, die dieser Abschlussarbeit angehängt wird.

b. Die Erstellunng eine interaktiven Lernmedien mit der Macromedia Flash Professional 8 zum Thema „Deutsche Geschichte” haben von der Experten evaluiert. Die Evaluation sind die Evaluation der Wörter und der Sprache der deutschen Untertitel, die von dem Muttersprachler gemacht wird und das Design wird von Experten des Designs gemacht.

Basierend auf der obigen Meinung kann geschlossen werden, dass die interaktiven Lernmedien mit der Macromedia Flash Professional 8 zum Thema „Deutsche Geschichte” gut sind und Inhaltlich ist das Thema sehr 
interessant, aber der Rechtschreibung und Großbuchstaben mussen beachtet werden.

\section{LITERATURVERZEICHNIS}

Anggara, Yuda. 2008. Membuat Gambar Vector Dan Atraktiv Dengan Macromedia Flash Professional 8. Bandung: Yrama Widya

Arsyad, A. 2002. Media Pembelajaran. Jakarta: Rajawali Pers

Arsyad, Azhar. 2011. Media Pembelajaran. Jakarta: PT. Raja Grafindo Persada Arsyad, Azhar. 2013. Media Pembelajaran. Jakarta: PT. Raja Grafindo Persada Aqib, Zainal. 2014. Model-Model, Media Dan Strategi Pembelajaran Kontekstual. (Inovatif). Bandung: Yrama Yuda

Barus dan Suratno. 2015. Pemanfaatn Candi Bahal Sebagai Media Pembelajaran Alam Terbuka Dalam Proses Belajar Mengajar. Medan: Perdana Mitra Handalan

Daryanto. 2010. Media Pembelajaran. Yogyakarta: Gava

Djamarah. 2006. Strategi Belajar Mengajar.Jakarta: RinekaCipta

Kusrianto, Adi. 2006. Panduan Lengkap Memakai Macromedia Flash Professional 8. Jakarta: PT. Elex Media Komputindo.

Luscher Renate, 2005. Von der Wende bis heute Landeskunde Deutschland. München, Deutschland Umschlagfoto: Fotolia

Mai Manferd, 2009. Deutsche Geschichte. Hamburg: Beltz Druckpartner

Rusman, 2012. Belajar dan Pembelajaran Berbbasis Komputer Mengembangkan Professionalisme Guru Abad 21. Bandung : Alfabeta

Sugyono. 2009. Metode Penelitian Bisnis (Pendekatan Kuantitatif, Kualitatif, dan $R \& D)$. Bandung:Alfabeta

Sukoco, dkk. 2014. Pengembangan Media Pembelajaran Interaktif Berbasis Komputer Untuk Peserta Didik Mata Pelajaran Teknik kendaraan Ringan. Jurnal Pendidikan Teknologi dan Kejuruan, 22(2) 215-225

Susilana, R dan Riyana. 2008. Media Pembelajarn. Bandung : CV Wacana Prima Tim Divisi Litbang Macdoms. 2006. Mahir Dalam Tujuh Hari Macromedia Flash Professional 8. Madiun: Andi 
Wahyono, Teguh. 2006. Etika Komputer Dan Tanggung Jawab Profesional Di Bidang Teknologi Dan Informasi. Andi: Yogyakarta https://isakabdillah.wordpress.com/author/isakabdillah/gelesen am 10. August 2017, um 14.00

http://www. Tatsachen Über Deutschland. Tatsachen_2015_deu.pdf gelesen am 10. August 2017, um 14.18 